\title{
Pro/con debate
}

\section{Pro: Access to advanced}

\section{therapies for severe asthma} should be restricted to patients with satisfactory adherence to maintenance treatment

Inhaled corticosteroids (ICS) are the core component of asthma treatment and the only maintenance therapy known to prevent asthma death. There is currently no evidence that biologics prevent asthma death in people with asthma, and as such, biologics cannot be recommended as an alternative to ICS therapy. Taking the time to assess adherence and provide interventions and education to support patients in asthma self-management has been shown to improve patient outcomes. It is therefore our responsibility as healthcare professionals to ensure that patients are supported, educated and motivated to adhere to ICS therapy before progressing to biologic therapies.
Asthma mortality rates have declined dramatically in most countries over recent decades, due to improved diagnosis and better treatment [1]. The place of inhaled corticosteroids (ICS) in managing asthma is not disputed. Enhanced understanding of asthma biology and particularly the role of type 2 inflammation has emphasised the value of ICS in improving asthma control and preventing asthma deaths [2]. They are the most effective antiinflammatory therapy for asthma, suppressing the expression and/or effects of multiple inflammatory molecules that are active in asthma. The very earliest studies of the efficacy of ICSs in asthma demonstrated their ability to reduce or eliminate the need for oral corticosteroids (OCSs) as a maintenance treatment [3], and even at low doses, they reduce the risk of severe asthma exacerbations and death from asthma [2]. Today, international guidelines recommend initiating ICS treatment in all patients with asthma due to the evidence of efficacy and safety [4].
Despite the benefits of ICS, a small minority of patients with severe asthma have disease which is refractory to high-dose ICS therapy and require additional OCS to achieve acceptable control of symptoms and exacerbations [5]. Although some estimates have suggested that severe asthma affects $5-10 \%$ of the total asthma population, it is well recognised that many patients prescribed high-dose ICS remain poorly controlled because of other modifiable factors. Such individuals fit in the classification schema of "difficult-to-treat" asthma rather than severe treatment refractory asthma. The underlying challenges of difficult-to-treat asthma usually relate to suboptimal adherence and selfmanagement skills, and unaddressed comorbidities, triggers and aggravating factors. Medication nonadherence, particularly to ICS, is a modifiable factor that is particularly prevalent in difficult-to-control asthma, with previous estimates of non-adherence of $\sim 50-65 \%$ by prescription refills $[6,7]$. Nonadherence in such patients has been associated with

@ERSpublications

ICS save lives. It is therefore our responsibility as healthcare professionals to ensure that patients are supported, educated and motivated to improve poor ICS adherence before treatment is further escalated to biologic therapy. https://bit.ly/3o2q26i
Cite as: Murphy AC, Boddy C, Bradding P. Pro: Access to advanced therapies for severe asthma should be restricted to patients with satisfactory adherence to maintenance treatment. Breathe 2021; 17: 210024 .

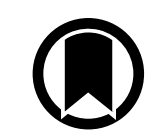

CrossMark 
adverse outcomes such as impaired lung function, increased eosinophilic airway inflammation, and an increased likelihood of having previously required critical care admission when compared with those who are adherent to ICS [7]. Furthermore, suboptimal ICS adherence is associated with an increased mortality from asthma [2]. Once poor adherence to medicines and sub-optimal inhaler technique has been addressed the true prevalence of patients who remain uncontrolled on high-dose inhaled ICS falls to $-3-4 \%[8,9]$, indicating that low adherence to ICS is a major factor in poor asthma control.

ICS save lives. It is therefore our responsibility as healthcare professionals (HCPs) to ensure that patients are supported, educated and motivated to improve adherence. Taking the time to assess adherence and provide interventions and education to support patients in self-management has been shown to improve patient outcomes. In 1987, a nationwide health survey in Finland showed that only $33 \%$ of Finnish asthma patients used ICS. This led to a strategy to improve asthma control that involved multiple methods, including adherence support and inhaler technique optimisation using decision support systems, and interactive education with patients [10]. By 1995, the ratio of ICS to $\beta_{2}$-agonists reached a ratio of 1.0 , with over $85 \%$ of patients using ICS daily. Data from the Finnish Asthma Programme confirmed that the increase in ICS prescribing was associated with a decline in asthma morbidity, reducing the utilisation of emergency services, hospitalisations and deaths.

Medicine adherence support should form a core component of all asthma services, from primary care through to tertiary specialist centres. However, there is still no agreed pathway on how non-adherence is best tackled. Assessing and supporting adherence remains a key challenge as it takes time and requires HCPs to develop a skill set that enables them to understand the patient's beliefs about asthma and its treatment, and to develop individualised strategies to support the patient. Non-adherence should not be seen as the patient's problem. It represents a fundamental limitation in the delivery of healthcare, often because of a failure to fully agree the prescription in the first place, or to identify and provide the support that patients need later on. Identifying non-adherence enables a personalised treatment approach and ensures that each individual is prescribed the right medicine at the right time. Without accurate adherence measurement, progression to a targeted high-cost biologic therapy may be inappropriate.

Five biologic therapies have approved indications for the treatment of severe asthma, targeting: 1) IgE (omalizumab, indicated for moderate-to-severe allergic asthma), 2) antiinterleukin (IL)-4R $\alpha$ (dupilumab, indicated for moderate-to-severe asthma with an eosinophilic phenotype or with OCS-dependent asthma), and
3) IL-5 (mepolizumab, reslizumab or the IL-5R $\alpha$ benralizumab, all indicated for severe eosinophilic asthma). All have a UK licence that states that high-dose ICS should be continued alongside their use. These targeted therapies have been shown to reduce asthma exacerbations, improve lung function, reduce OCS use, and improve quality of life in appropriately selected patients. Although they have the potential to reduce asthma mortality this has not been demonstrated. There is certainly no evidence in people who are nonadherent to ICS that biologic therapy would reduce asthma mortality, whereas it is clear that improving adherence to ICS does. The clinical trials that proved the efficacy and safety of these biologics were similar in their inclusion criteria, study designs, and measured outcomes [11]. It is important to recognise that in clinical trials, patient care is carefully optimised with patients both encouraged and motivated to use moderate-to-high dose ICS regularly; this is evidently profoundly different to real-life practice, where non-adherence is common. It cannot be assumed that biologics are effective when inadequate doses of ICS are taken. So, while it has been argued by some that nonadherent patients at high risk of asthma death should be started on biological therapies as a priority to reduce risk [12], there is no evidence to support this.

The current evidence on the relationship between ICS adherence and clinical outcomes in those receiving biologics is inconsistent. D'ANCONA et al. [13] highlighted there was an increased risk of exacerbations and reduced probability of stopping OCS in those people receiving mepolizumab who were deemed non-adherent to ICS compared with an adherent cohort. The authors suggest that the clinical response to mepolizumab is poor in people who are non-adherent to ICS. Interestingly, a further small observational study by D'ANCONA et al. [14] showed that patients non-adherent to ICS therapy had similar outcomes after 12 months of benralizumab to those who were deemed to be adherent. The inconsistent results may be attributable to the biologic administered, but both studies have a number of important limitations, which increase the vulnerability to biases, potentially explaining the dichotomy of the results. Both studies were small and retrospective in design and measured adherence using the medication procession ratio (MPR), a function of prescriptions issued rather than a direct measure of medication use. A review of prescription refill data alone does not detect non-intentional non-adherence due to poor inhaler technique that can develop rapidly after inhaler training [15]. Also, it assumes that the drugs are always used. There is no way to tell if the patient shared, stockpiled or dumped the medications after collecting a prescription. Further research is required to understand the synergy of ICS and biologic therapies, but it is clear ICS exert effects on the airway well beyond those of targeted therapies. 
The known significant protective effects of an ICS make treatment optimisation an essential intervention; ignoring this in favour of escalating treatment to biologic therapy is without evidence, potentially increases treatment costs and is possibly hazardous. The inappropriate use of biologics increases the financial burden on the UK National Health Service and reduces capacity to deliver these drugs in severe asthma centres. It also exposes patients to a medication that in the longterm may have side-effects that are not yet known about. Although the biologic agents approved for asthma appear safe, interfering with the delicate intricacies of the immune system raises some safety concerns, and long-term safety data is lacking for most of them.

Identifying non-adherence is the first step to tackling it, and review of prescription refills in primary care is a relatively easy first step to detecting this. For some patients, detection, discussion and subsequent review in primary care will be all that is needed, but currently this is underused as demonstrated by the high rates of ICS nonadherence identified by prescription refill rates in people with asthma referred to secondary care [6, 7].

Combining prescription refill data with objective exhaled nitric oxide fraction $\left(F_{\mathrm{ENO}}\right)$ testing can identify non-adherent patients who fill prescriptions but do not actually take their medication, either intentionally or unintentionally. In addition, serial $F_{\text {ENO }}$ measurements can be used to drive improved adherence to current ICS therapy in clinical practice [16]. A $F_{\mathrm{ENO}}$ value of $>45 \mathrm{ppb}$ is a strong predictor of ICS non-adherence and has led to the concept of $F_{\mathrm{ENO}}$ suppression testing, where a fall in the $\triangle \log _{10} F_{\mathrm{ENO}}$ value of $\geq 0.24$ (approximately a $42 \%$ fall in $F_{\mathrm{ENO}}$ ) with directly observed ICS therapy defines non-adherence (these patients are referred to as $F_{\text {ENO }}$ suppressors) [17]. The use of an electronic monitoring device, such as the inhaler compliance assessment (INCA) device, that measures both inhaler use and technique, is a useful tool for the identification and management of persistent ICS non-adherence, and removes the extensive resources required for directly observed therapy [17].

HEANEY et al. [18] demonstrated that electronic monitoring of ICS use for 7 days using the INCA device attached to a fluticasone Accuhaler, in addition to the patient's usual medication and coupled with patient home $F_{\mathrm{ENO}}$ measurements, identified a group of difficult-to-control asthma patients presenting with a $F_{\mathrm{ENO}}>45 \mathrm{ppb}$ who were ICS responsive and suppressed their $F_{\mathrm{ENO}}(>42 \%$ fall) when using monitored therapy. $\sim 65 \%$ were non-adherent by this metric, similar to the rates identified by prescription refill monitoring [7]. Subsequent monitoring for 28 days maintained the fall in $F_{\mathrm{ENO}}$, and was accompanied by significant improvements in lung function, Asthma Control Questionnaire and blood eosinophil counts. Thus, $65 \%$ of people with difficult-to-control asthma presenting with $F_{\mathrm{ENO}}>45 \mathrm{ppb}$ can be controlled with improved ICS adherence, and potentially do not need to progress to biologic therapy.

The objective data from INCA monitoring can be used in an adherence discussion with patients. In a recent real-life service evaluation, BoDdy et al. [19] used the INCA monitoring device, alongside monthly appointments with a specialist nurse, to help identify and manage ICS non-adherence in difficult-to-control asthma. Again, in people presenting with a $F_{\mathrm{ENO}}>45 \mathrm{ppb}, 65 \%$ suppressed their $F_{\mathrm{ENO}}$ and were classified as non-adherent. Only $35 \%$ of $F_{\mathrm{ENO}}$ suppressors progressed to biologic therapy compared to $73 \%$ of $F_{\mathrm{ENO}}$ non-suppressors. 13 of the $F_{\mathrm{ENO}}$ suppressors who did not progress to biologic therapy were using OCS with a median baseline dose of $10 \mathrm{mg}$ prednisolone daily, and this was successfully reduced to a median of $3 \mathrm{mg}$ daily without a loss of asthma control. Further dose reductions were limited predominantly by adrenal suppression. These findings suggest that it is possible to intervene and address modifiable factors influencing ICS adherence in a substantial proportion of people with asthma. This nurseled adherence support is likely to be very costeffective. If the patients not receiving biologics had progressed to these, the drug costs alone would be approximately GBP 400000 per annum at present.

Many patients with a history of non-adherence are likely to require ongoing adherence support throughout their treatment pathway and even if they do improve their adherence before starting a biologic, it must not be assumed that patients will remain adherent when receiving biologic treatment. Non-adherence should be seen as a variable behaviour: adherence rates vary not just between individuals but within the same person over time and across treatments. The majority of severe asthma centres are moving patients to selfadministration of biologic therapies at home, akin to biologic delivery in other long-term conditions. As we know many patients with asthma either forget to use their ICS or decide it is not necessary when their asthma is well-controlled, and this may also apply to their biologic therapy too.

This has been demonstrated in other diseases where biologics have been prescribed for several years. A recent study by THORNELOw et al. [20], assessed "real-world" levels of self-reported non-adherence to conventional and biologic systemic therapies used for psoriasis. The study demonstrated that overall $22.4 \%(n=811)$ of the patients were classified as "non-adherent" (12\% intentionally and $10.9 \%$ unintentionally), with $16.4 \%$ specifically non-adherent in using subcutaneous biologics. A further study assessing adherence to subcutaneous anti-tumour necrosis factor therapy [21] reported that $27 \%$ of patients were non-adherent to biologic therapy at least once within the first 6-month period with associated worse treatment outcomes and higher healthcare costs in that cohort. Such behaviour changes will need to be considered in people with severe asthma 
with adherence monitored regularly through the measurement of appropriate biomarkers.

\section{Summary}

- ICS are the core component of asthma treatment and the only maintenance therapy known to prevent asthma death.
- ICS adherence must be assessed in all healthcare settings, and ongoing support provided. This applies equally to patients starting biologic therapy, and once a patient is established on a biologic therapy this approach cannot be relaxed.

- There is currently no evidence that biologics prevent asthma death in people with asthma, and as such, biologics cannot be recommended as an alternative to ICS therapy.

\section{Affiliations}

\section{Anna C. Murphy' ${ }^{1}$ Claire Boddy ${ }^{1}$, Peter Bradding ${ }^{1,2}$}

${ }^{1}$ Dept of Respiratory Medicine, University Hospitals of Leicester NHS Trust, Glenfield Hospital, Leicester, UK. ${ }^{2}$ Dept of Respiratory Sciences, Institute for Lung Health, University of Leicester, Glenfield Hospital, Leicester, UK.

\section{Conflict of interest}

A.C. Murphy has no conflicts of interest. C. Boddy reports personal fees from Sanofi Genzyme (conference attendance fees), personal fees and other from Teva (travel and attendance fees for attendance at specialist nurse meetings), outside the submitted work. P. Bradding reports other from Chiesi (attendance at scientific meeting), other from Genentech-Roche (consultancy via the University of Leicester), other from Boehringer-Ingelheim (consultancy via the University of Leicester), other from Teva (attendance at scientific meeting) and other from Sanofi (attendance at scientific meeting), outside the submitted work.

\section{References}

1. Wijesinghe $M$, Weatherall M, Perrin K, et al. International trends in asthma mortality rates in the 5- to 34-year age group: a call for closer surveillance. Chest 2009; 135: 1045-1049.

2. Suissa S, Ernst P, Benayoun S, et al. Low-dose inhaled corticosteroids and the prevention of death from asthma. N EnglJ Med 2000; 343: 332-336.

3. O'Byrne P, Fabbri LM, Pavord ID, et al. Asthma progression and mortality: the role of inhaled corticosteroids. Eur Respir 2019; 54: 1900491

4. Global Initiative for Asthma (GINA). Global strategy for asthma management and prevention report. 2020. www.ginasthma.org Date last accessed: 14 January 2021.

5. Chung KF, Wenzel SE, Brozek JL, et al. International ERS/ATS guidelines on definition, evaluation and treatment of severe asthma. Eur Respir J 2014; 43: 343-373.

6. Gamble J, Stevenson M, McClean E, et al. The prevalence of nonadherence in difficult asthma. Am J Respir Crit Care Med 2009; 180: 817-822.

7. Murphy AC, Proeschal A, Brightling CE, et al. The relationship between clinical outcomes and medication adherence in difficult-to-control asthma. Thorax 2012; 67: 751-753.

8. Hekking P-PW, Wener RR, Amelink M, et al. The prevalence of severe refractory asthma. J Allergy Clin Immunol 2015; 135: 896-902.

9. Blake KV. Improving adherence to asthma medications: current knowledge and future perspectives. Curr Opin Pulm Med 2017; 23: $62-70$.

10. Haahtela T, Tuomisto LE, Pietinalho A, et al. A 10 yea asthma programme in Finland: major change for the better Thorax 2006; 61: 663-670.

11. Doroudchi A, Pathria M, Modena BD. Asthma biologists: comparing trial designs, patient cohorts and study results. Ann Allergy Asthma Immunol 2020; 124: 44-56.

12. Green RH, Shaw D. Strict adherence rules to obtain monoclonal therapy might cost lives. Lancet Respir Med 2017; 5: 678-679.
13. d'Ancona G, Kavanagh J, Roxas C, et al. Adherence to corticosteroids and clinical outcomes in mepolizumab therapy for severe asthma. Eur Respir J 2020; 55: 190225.

14. d'Ancona G, Kavanagh JE, Dhariwal J, et al. Adherence to inhaled corticosteroids and clinical outcomes following a year of benralizumab therapy for severe eosinophilic asthma. Allergy 2021; in press [https://doi.org/10.1111/all.14737].

15. Van der Palen J, Klein J, Kerkhoff $\mathrm{AH}$, et al. Evaluation of the long-term effectiveness of three instruction modes for inhaling medicines. Patient Educ Couns. 1997; 32: S87-S95.

16. Price, D, Ryan, D, Burden, A, et al. Using fractional exhaled nitric oxide (FeNO) to diagnose steroid-responsive disease and guide asthma management in routine care. Clin Transl Allergy 2013; 3: 37

17. McNicholl DM, Stevenson M, McGarvey LP, et al. The utility of fractional exhaled nitric oxide suppression in the identification of nonadherence in difficult asthma. Am J Respir Crit Care Med 2012; 186: 1102-1108

18. Heaney LG, Busby J, Bradding P, et al. Remotely monitored therapy and nitric oxide suppression identifies nonadherence in severe asthma. Am J Respir Crit Care Med 2019; 199: 454-464.

19. Boddy CE, Naveed S, Craner M, et al. Clinical outcomes in people with difficult-to-control asthma using electronic monitoring to support medication adherence. J Allergy Clin Immunol Pract 2020; 9: 1529-1538.

20. Thornelow RJ, Griffiths CE, Emsley R, et al. Intentional and unintentional medication non-adherence in psoriasis: the role of patients' medication beliefs and habit strength. J Invest Dermatol 2018; 138: 785-794.

21. Bluett J, Morgan C, Thurston L, et al. Impact of inadequate adherence on response to subcutaneously administered anti-tumour necrosis factor drugs: results from the Biologics in Rheumatoid Arthritis Genetics and Genomics Study Syndicate cohort. Rheumatology (Oxford) 2015; 54: 494-499. 